\title{
8
}

\section{Du fœtus au jeune enfant : sentir, regarder, comparer, grandir - De la variabilité des adaptations}

> D'où vient l'énergie qui permet au bébé in utero de grandir si vite?

> Le bébé et la mère ont-ils la même composition en acides gras ?

> Est-il vrai que le cerveau est très gras ?

> Le cerveau d'un enfant grandit-il au même rythme que l'enfant?

> Pourquoi le lait maternel est-il si riche en lipides ?

> Comment ont été formulés les laits maternisés ?

> Les laits maternisés d'aujourd'hui sont-ils différents de ceux des années 1970 ?

> Il mange comme quatre, son frère a un appétit d'oiseau et les deux grandissent au même rythme. Pourquoi ?

> Faut-il ou non réduire la consommation de lipides chez le jeune enfant? 
Une grande partie des systèmes complexes précédemment présentés prennent vie avec l'enfant. À ce titre, l'étude de la vie fotale, puis des premières années de l'enfant, représente une mise en application de toutes les leçons précédentes... et une leçon d'humilité quant à la complexité des systèmes de régulation retenus par l'évolution.

\section{LA VIE FCETALE}

"Cela fait quatre mois que j'attendais. Mais ce matin, cette série de coups de pied, en rafale, c'était bien toi, mon bébé! J'ai enfin pu te voir à l'échographie. Calme, tu suçais ton pouce, avec une sérénité impressionnante. Et pourtant, quatre mois encore avant que je ne te tienne dans mes bras, mes mains... »

Alors qu'il ne lui reste que 4 mois de vie in utero avant sa naissance, le futur bébé a un programme chargé : $2,5 \mathrm{~kg}$ à prendre, $40 \mathrm{~cm}$ à gagner d'ici sa naissance. Un planning très chargé si l'on y regarde de plus près : par exemple, grandir de $40 \mathrm{~cm}$ en 4 mois, cela équivaut à un rythme de croissance de 1,20 m par an. Quels sont les mécanismes expliquant qu'un tel programme puisse être tenu?

\section{D'où vient l'énergie nécessaire?}

Une telle croissance nécessite une production énorme d'énergie. Tout est délirant! Prenons l'exemple de ces simples coups de pied. Un gaspillage ? Non, bien au contraire : il s'agit d'un entraînement, d'une mise en place. Pour autant, à l'énergie nécessaire aux mouvements du bébé in utero s'ajoutent les besoins liés à la croissance, au développement harmonieux des organes. Aussi, d'où vient cette énergie : des protéines ? Des glucides ? Ou des lipides?

À cet âge, l'utilisation, sur le plan énergétique, des protéines est inexistante. Trop précieuses pour la croissance, elles ne sont pas brûlées pour fournir de l'énergie. L'utilisation des glucides est trop limitée : nous ne faisons pas partie du monde végétal mais du monde animal, et le bébé en gestation connaît, nous l'avons vu, une période 
de croissance explosive. Or 1 gramme de glucides apporte « seulement » 4 calories... quand 1 gramme de lipides en offre 9 ! L'équation est vite résolue : les lipides représentent la source majeure d'énergie durant les quatre derniers mois de gestation.

\section{L'acide palmitique, élément clé}

À cet âge de la vie, le moteur énergétique est représenté par les lipides. $\mathrm{Ou}$, pour être plus précis, un membre spécifique de la grande famille des lipides : l'acide palmitique C16:0.

L'acide palmitique n'est ni plus ni moins que le carrefour, la plateforme de régulation énergétique, l'élément clé. Et pourtant, pendant longtemps, il est demeuré un mystère. Il faudra attendre l'accès au prélèvement sanguin, au niveau du cordon ombilical, in utero, et ce, pour une raison vitale (le diagnostic de la toxoplasmose congénitale) pour commencer à analyser les acides gras circulants à la $22^{\mathrm{e}}$, à la $32^{\mathrm{e}}$ et à la $37^{\mathrm{e}}$ semaine de gestation chez la femme. Ces travaux furent réalisés par Madame P. Crastes de Paulet et P. Sarda dans les années 1985.

L'acide palmitique et l'acide palmitoléique (dérivé du premier, qui compte également 16 atomes de carbone mais possède une insaturation sur le septième carbone) sont les acides gras énergétiques circulants les plus importants.

\section{Une nécessaire régulation}

Une question se pose néanmoins : les taux d'acide gras palmitique (C16:0) circulant dans le cordon ombilical s'avèrent très élevés, plus élevés qu'à toute autre période de la vie. À ce même moment de la vie gestationnelle, les taux d'acide gras palmitoléique (C16:1 n-7) se révèlent également très conséquents : $6,5 \%$ des acides gras à la $32^{\mathrm{e}}$ semaine, $5,6 \%$ à la $37^{\mathrm{e}}$ semaine... contre $3,4 \%$ chez la femme enceinte et $2,5 \%$ chez la femme n'attendant pas d'enfant. Il semble donc exister une régulation très importante, permettant au bébé dans le ventre de sa mère de disposer de taux très élevés d'acide palmitique, tout en bénéficiant d'une réserve d'acide palmitoléique. 
Quel est l'intérêt d'une telle situation ? Permettre à l'organisme du bébé de fabriquer tous les lipides dont il a besoin par lipogenèse de novo. Une telle situation ne se rencontre plus que dans une situation pathologique grave : l'insuffisance pulmonaire de la mucoviscidose, où l'on observe des taux d'acide palmitoléique atteignant $6,3 \%$ des acides gras circulants.

\section{Variations pondérales de la mère}

Le problème énergétique des derniers mois de croissance gestationnelle semble ainsi résolu, si l'on ajoute les variations pondérales de la mère. Celles-là ne devraient pas dépasser $10 \mathrm{~kg}$ pour un bébé de $3,5 \mathrm{~kg}$ à la naissance, auxquels s'ajoutent $1,5 \mathrm{~kg}$ d'annexes (placenta, etc.) et de l'ordre de $5 \mathrm{~kg}$ de réserves qu'elle perdra en trois mois avec l'allaitement, etc. Il faut néanmoins être très prudent.

En effet, manifestement, une partie de la variation de ce poids est régulée par une interaction entre le bébé et sa mère. Il en est ainsi par exemple du taux d'acide palmitoléique chez la femme enceinte, très différent de celui de la femme n'attendant pas d'enfant. L'exemple flagrant de mères déportées pendant la Seconde Guerre mondiale, affamées, ne pesant plus que $45 \mathrm{~kg}$ mais accouchant de bébés de $3,5 \mathrm{~kg}$, souligne le fait qu'il existe des régulations. Et les contre-exemples de telles naissances montrent que ce n'est pas toujours le cas...

\section{De l'énergie aux composants structuraux}

Le problème de l'apport d'énergie résolu, reste la question de la synthèse des structures. Autrement dit, la transformation de cette " énergie quantitative ( (le volume d'énergie nécessaire) en « énergie qualitative » (les bons lipides au bon endroit). Pour ce faire, l'organisme dispose, nous l'avons vu, de deux types d'outils :

- les élongases qui permettent d'ajouter, deux à deux, des atomes de carbone à la chaîne carbonée des acides gras et ainsi de l'allonger ; 
- les désaturases qui, par un mécanisme de déshydrogénation, remplacent une simple liaison par une double liaison. Chaque désaturase est spécifique : elle ne peut agir que sur le carbone situé à une distance définie du groupement carboxyle $\mathrm{COOH}$. Ainsi, la delta-9 désaturase ne peut agir par exemple que sur le neuvième carbone.

Dès lors, au moyen de ces deux outils, l'organisme peut fabriquer un très grand nombre d'acides gras différents. Un très grand nombre... mais pas tous, car les Mammifères ne disposent pas de toutes les désaturases. Ainsi, les acides gras de la famille des oméga-3 (insaturation située sur le troisième carbone à partir du groupement méthyle $\mathrm{CH}_{3}$ ) et des oméga-6 (insaturation située sur le sixième carbone à partir du groupement méthyle $\mathrm{CH}_{3}$ ) ne peuvent être fabriqués qu'à partir de leurs précurseurs à 18 atomes de carbone : l'acide alpha-linolénique (C18:3n-3) et l'acide linoléique (C18:2 n-6).

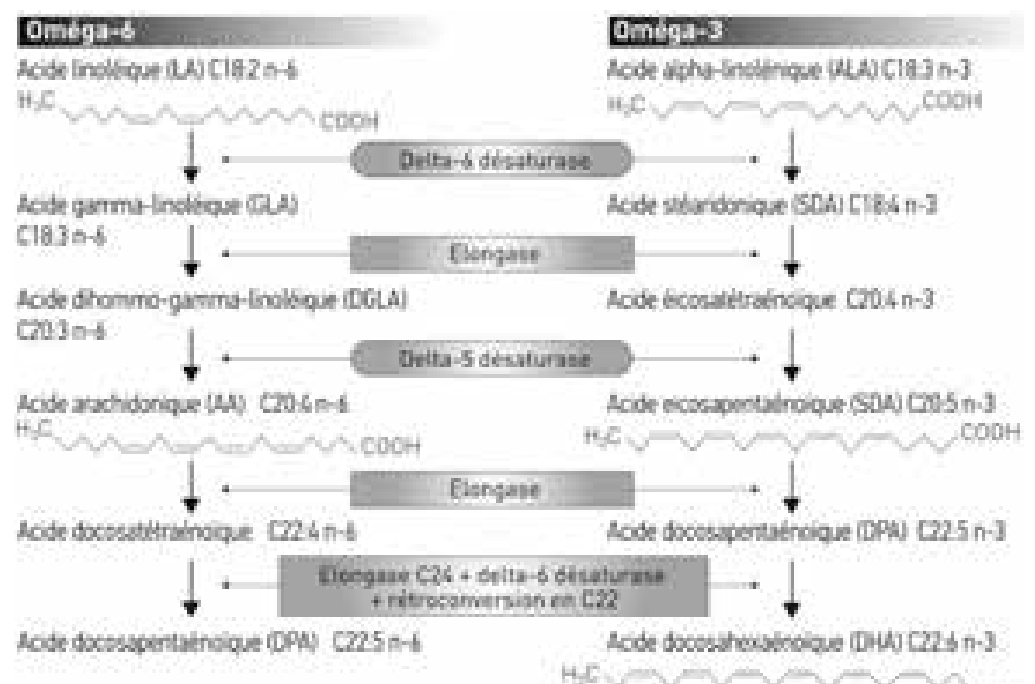




\section{Nouveau-né : un cerveau et une peau riches en lipides}

À la naissance, le cerveau du bébé pèse $350 \mathrm{~g}$, soit de l'ordre d'un quart du poids d'un cerveau adulte. Il est constitué en majorité d'eau $(320 \mathrm{~g}$ sur $350 \mathrm{~g})$ et, dans une moindre mesure, de lipides $(9 \mathrm{~g})$. Autre « zone » très riche en lipides chez le nouveau-né : la peau, puisque la totalité de la graisse cutanée du bébé représente de l'ordre de $350 \mathrm{~g}$. Soit autant de graisses dans le cerveau que dans la peau.

\section{Un système complexe}

Penchons-nous justement sur un de ces acides gras polyinsaturés de la sous-famille des oméga-3 : le DHA (acide docosahexaénoïque C22:6 n-3), l'acide gras le plus long (22 atomes de carbone), «l'acide polyinsaturé du cerveau ».

La découverte du DHA en 1966 (F. Mendy et P. Lemarchal) déclencha une double polémique. Ainsi, son identification comme du C22:6 $n$-3 (donc un acide gras porteur de six insaturations), et non comme du DPA (C22:5 n-3, porteur de cinq insaturations), fut l'objet d'une première controverse, qui n'a été finalement éclaircie que récemment : le jeu des désaturases et élongases aurait pu laisser croire que l'accroissement de l'apport alimentaire en acide alpha-linolénique (C18:3n-3,6,9), cet oméga-3 à 18 atomes de carbone d'origine végétale indispensable aux Mammifères, rimerait avec une augmentation du DHA, un de ses dérivés. Autrement dit, l'idée simple selon laquelle plus on augmente la quantité d'acide gras au début de la chaîne, plus on " récupère " d'acides gras au bout de cette chaîne de réactions... À la manière d'une usine qui produit plus de produits finaux quand on augmente la quantité de matière première.

Pourtant, il n'en est rien. Cette série de désaturations et d'élongations qui aboutit au produit "phare ", le DHA, est extrêmement régulée. À cet âge de la vie, l'objectif absolu est d'éviter toute augmentation de l'EPA (acide eicosapentaénoïque, C20:5 n-3) au-dessus du «presque zéro ». En biologie humaine, à condition d'éviter au 
maximum l'apport exogène d'EPA, cette étape est immédiatement franchie. Un nouveau paradigme se dessine : l'acide alpha-linolénique (oméga-3 végétal) et son dérivé le DPA (C20:5 n-3) endogène, réserve potentielle et source du DHA de régulation très différente, constitue un ensemble très régulé d'importance majeure.

Seconde polémique : les teneurs en acide linoléique (oméga-6) et en acide alpha-linolénique (oméga-3) du sang circulant au niveau du cordon ombilical à la naissance se révèlent plus faibles que celles retrouvées chez la mère à la naissance, alors que les teneurs en acide arachidonique (un oméga-6 dérivé de l'acide linoléique), en DPA et en DHA (deux oméga-3 dérivés de l'acide alpha-linolénique) sont plus élevées.

Cela conduisit à d'importantes controverses : certains y voyaient la preuve de la non-essentialité des acides linoléique et alpha-linolénique chez le nourrisson (puisqu'il y en a moins dans le cordon que dans le sang de la mère), puis les rôles exclusifs des dérivés de l'acide arachidonique et du DHA.

La vérité, démontrée lentement depuis, s'avère à la fois plus simple et complexe : l'organisme humain a la possibilité de modifier, d'allonger les acides gras " parents » (à savoir les acides linoléique et alpha-linolénique) en dérivés supérieurs selon des mécanismes rigoureusement contrôlés et en adéquation stricte avec ses besoins. Or, ces acides gras polyinsaturés, allongés et modifiés, se révèlent nettement plus oxydables : véritables bombes à retardement, ils doivent être encadrés, neutralisés, protégés et dirigés uniquement vers la destination utile. Cette régulation est strictement coordonnée avec le métabolisme des « acides gras parents ». Il y a toujours continuité, et non rupture et indépendance, entre les acides gras parents et les dérivés supérieurs. Nous sommes en présence d'un système extrêmement régulé, et cette régulation persistera tout au long de la vie.

La démonstration de l'efficacité de ce système a été apportée par des études de développement au cours de la grossesse et de l'allaitement chez des végétariens (aucune consommation de viande) et des 
végétaliens (aucune consommation de viande ni de produits animaux comme les œufs, le lait, etc.). Elles ont souligné que seuls les acides gras indispensables doivent être obligatoirement présents dans l'alimentation.

Ainsi, seuls les acides gras indispensables, à savoir l'acide linoléique et l'acide alpha-linolénique, d'origine végétale, doivent être obligatoirement présents dans l'alimentation.

Il reste de nombreuses inconnues dans la régulation endocrinométabolique de la grossesse, mais les quelques lois générales qui ont émergé sont d'une importance capitale. Elles définissent les possibilités extrêmes des régulations que le duo formé par la mère et son bébé peut mettre en jeu. La possibilité de ces régulations extrêmes reste enfouie quelque part, parfois réactivable dans certaines situations limites chez l'Homme : phénomène de "catch-up » (ou croissance compensatrice endogène, qui fait suite à une période de restriction alimentaire), défaillances pulmonaires aiguës ou chroniques (une gangue de calcaire liée à une péricardite tuberculeuse empêche le cœur de grandir, l'organisme fonctionne alors en régime minimum ; la seule suppression des plaques suffit à un rattrapage du retard cumulé).

Encore insuffisamment explorée, cette zone d'hyperanabolisme que représente la grossesse est le plus bel exemple des possibles extrêmes que recèle la physiologie humaine. Tout amalgame à partir d'études menées chez l'animal sera source d'erreurs considérables. Il faut revenir à une observation longue et approfondie des rares données progressivement à notre portée, irréfutables, et aux conséquences ouvrant le champ des possibles en physiologie humaine.

\section{LE DÉVELOPPEMENT DU JEUNE ENFANT}

À la naissance, un nouveau-né mesure de l'ordre de 50 centimètres, pèse entre 3 et 3,5 kilos. Le poids de son cerveau équivaut au quart du poids d'un cerveau d'adulte, soit 350 grammes. 
La prochaine étape de son développement va durer six ans. Six années au terme desquelles l'enfant mesurera de l'ordre de 1,14 mètre, pèsera environ 20 kilos, mais surtout dont le cerveau représentera $9 / 10 \mathrm{du}$ poids d'un cerveau d'adulte, soit 1230 grammes. En réalité, la croissance du cerveau sera achevée à 6 ans. Par la suite, le gain de poids proviendra essentiellement de l'enrichissement infini en connexions et synapses (zone de contact fonctionnelle qui s'établit entre deux neurones). Une étape intermédiaire majeure est à noter : à 3 ans, la masse du cerveau représente déjà les trois quarts de celle d'un cerveau d'adulte. Comment se déroule cette extraordinaire évolution?

\section{Le lait maternel, «la " référence}

Par chance, et c'est une situation unique en nutrition humaine, nous disposons d'un modèle de comparaison, écrasant toutes les études chez l'animal et ses dangereux amalgames : la croissance et l'évolution de l'enfant sous allaitement maternel, jusqu'à 2 ans dans certaines civilisations. Certes, l'allaitement maternel peut être presque parfaitement remplacé par des produits dérivés du lait de vache et additionnés d'acides gras essentiels ou indispensables d'origine végétale. Pour autant, le modèle directeur reste et demeure impérativement le lait maternel. Le lait maternel s'impose comme « la » référence et le combat doit être permanent pour éviter les dérives provenant d'une conception influencée soit par les modes, souvent très variables, de l'alimentation des adultes (le régime végétalien, le bio, etc.), soit par l'étude de l'évolution d'animaux de laboratoire, totalement différents quant à la place relative occupée par le rapport entre le poids du cerveau et le poids du corps.

À la naissance, la consommation spontanée du nourrisson allaité au sein représente 125 calories par kilo (contre $30 \mathrm{kcal} /$ $\mathrm{kg}$ chez l'adulte), dont $40 \%$ proviennent des lipides, $50 \%$ des glucides (essentiellement le lactose du lait, absence de fructose) et $10 \%$ maximum des protéines (plutôt 7 à $8 \%$ ). Cet apport 
lipidique s'avère considérablement plus élevé que ce qui fut longtemps considéré comme l'apport physiologique chez l'adulte, à savoir un apport de 1 gramme de lipides par kilo (et donc de 70 grammes de lipides par jour pour un adulte de 70 kilos) : le lait maternel apporte 5,55 grammes de lipides pour 1 kilo chez le nourrisson, soit bien plus que le fameux 1 gramme pour 1 kilo chez l'adulte. Et pourtant, il faut bien admettre que le nourrisson fait partie de la race humaine et que nous sommes face à une continuité de la naissance à l'âge adulte, puis aux abords du grand âge...

L'étude du lait maternel nous a également appris la très grande intrication dans le monde animal des apports lipidiques et protéiques. Toute lipogenèse de novo (création de lipides) à partir des protéines doit être limitée, dans la mesure du possible, en biologie humaine. Réservée aux situations extrêmes, elle n'est pas souhaitable en situation normale.

Les protéines du lait maternel, en coordination avec les lipides, représentent ainsi un remarquable exemple de gestion efficace. Moins de $10 \%$ de l'apport calorique total est apporté sous forme de protéines, pourtant nécessaires au moment de la croissance la plus intense ! La croissance s'effectue avec très peu de protéines et beaucoup de lipides.

\section{Évolution de la composition du cerveau}

En un demi-siècle, lentement, des connaissances ont été acquises sur la composition du cerveau à des âges clés, par exemple via le cumul d'autopsies faisant suite à des accidents. Ainsi, à l'âge clé de 140 semaines après la naissance, soit 2,5 ans, le poids du cerveau correspond, nous l'avons vu, à trois quarts du poids d'un cerveau adulte. Sa composition est rappelée en encadré. 


\section{Bilan de la composition du cerveau par ordre d'importance quantitative}

$\mu \mathrm{mol} /$ cerveau frontal

Acide oléique (18:1 $n-9)$

25000

Acide arachidonique (20:4 $n-6)$

12000

DHA (docosahexaénoïque) (22:6 $n-3)$

10000

Acide adrénique (22:4 $n$-3)

8500

Acide nervonique (24:1 n-9)

5000

DPA (docosapentaénoïque) (C22:5 n-3)

$>100$

EPA (eicosapentaénoïque) (C20:5 $n$-3)

Proche de 0

Il est important de retenir les proportions relatives plutôt que les chiffres précis. Ces données permettent de constater le délire que constitue la supplémentation de l'alimentation du nourrisson en huiles de poisson (dans lesquelles, en général, le ratio EPA/DHA est supérieur ou égal à 1), alors que l'organisme du nourrisson fait tout pour réduire le taux d'EPA à 0 . En revanche, une petite quantité de DPA est conservée. Seul le DHA pur, ou très dominant, devrait pouvoir être ajouté dans le cas exceptionnel où il serait nécessaire, par exemple dans le cas d'une grande prématurité (et cela à des doses inférieures à $20 \mathrm{mg} /$ jour$)$.

À la naissance, le corps de l'enfant contient 1 gramme de DHA, correspondant à la somme de toutes les réserves de constitution. L'énorme croissance du cerveau de l'enfant de sa naissance à ses 1 an requiert 6 grammes de DHA, soit 16,5 milligrammes par jour. Face à ces chiffres, on ne peut qu'être étonné des supplémentations recommandées chez l'adulte pour le seul «entretien » de son cerveau (pas de croissance chez l'adulte) : de 500 milligrammes à 6 grammes, voire 10 grammes par jour... soit 30 à 600 fois plus que les besoins d'un nourrisson dont le cerveau est, quant à lui, en pleine croissance! Sans parler du fait qu'il s'agit en général d'un mélange d'EPA et de DHA... 


\section{Formulation des laits maternisés}

Jusque dans les années 1960-1970, exception faite du lait maternel, les laits pour nourrissons étaient constitués le plus souvent de laits demi-écrémés sucrés, riches en protéines et en glucides, plutôt pauvres en lipides, semi-carencés en acides linoléique et linolénique. Pour des raisons éthiques évidentes, l'étude de tels laits chez le nourrisson serait interdite de nos jours. Mais entre 1960 et 1970, c'était l'alimentation habituelle des nourrissons nourris avec des laits industriels, dits de substitution. Il devenait indispensable de comparer le lait maternel avec des laits de substitution, sur le plan des lipides, grâce à des techniques nouvellement apparues (mises au point par Ackman en 1963) de chromatographie en phase gazeuse, qui permettent de séparer les molécules d'un mélange et donc d'en identifier la composition.

\section{Du seuil critique de Holman au rapport acide eicosatriénoïque/ acide arachidonique}

Préalablement, des études avaient été menées par les scientifiques Hansen et Holman, en 1963, auprès de 428 nourrissons, avec des résultats moyens. Seuls 30 nourrissons, en 1964, avaient été étudiés sur le plan biochimique, avec des techniques peu performantes, montrant :

- une chute du taux sérique de deux acides gras à C18 ou C20 atomes de carbone, en l'occurrence les acides gras diénoïque (deux doubles liaisons) et tétraénoïque (quatre doubles liaisons).

- une élévation du taux sérique des acides gras triénoïques (20 atomes de carbone, trois doubles liaisons), caractéristiques du syndrome de carence en acides gras essentiels.

Un seuil critique, dit de Holman, fut alors défini : il correspond à un rapport acides gras triènes/acides gras tetraènes (acides gras à trois doubles liaisons dont le taux s'est élevé durant l'expérience/acides gras à quatre doubles liaisons dont le taux s'est effondré) supérieur à 0,4 à 15 jours, puis atteignant 0,8 à 90 jours.

L'utilisation de chromatographies en phase gazeuse permit alors à un groupe de pédiatres français dont je faisais partie (A. Rossier, 
F. Alizon, P. Verger et F. Mendy) de compléter et valider, sur le plan scientifique, le syndrome de la carence en acide linoléique : le rapport acides gras triènes/acides gras tétraènes devint le rapport C20:3n-9/ C20:4 n-6 (acide eicosatriénoïque/acide arachidonique). L'étude porta au total sur 102 prématurés et nourrissons, répartis sur 123 périodes, et sur l'analyse de 205 chromatogrammes.

À 15 jours de vie, le rapport (C20:3n-9)/(C20:4n-6) était de 0,1 (et non de 0,4 comme établi par Hansen et Holman) ; très rapidement, il chuta à des valeurs inférieures à 0,04 , puis à des taux encore plus bas au bout du $115^{\mathrm{e}}$ jour de l'étude, avec les laits maternisés comme avec le lait maternel.

L'ensemble fut rapporté, après plusieurs articles intermédiaires dans la presse médicale ou scientifique, au sein d'une publication dans Archives des sciences physiologiques, en 1970, sous le titre: "Variations du rapport C20:3 n-9/C20:4 n-6 dans le sérum de nourrissons suivant l'âge et le régime ${ }^{132}$. Ce travail fut considéré pendant plusieurs années comme l'article de référence. D'où la surprise de voir réapparaître récemment, sous la signature d' " officiels de la nutrition », le vieux rapport d'Holman. Pourquoi déterrer ce rapport obsolète, réévalué depuis ? Il semblerait que l'objectif poursuivi par les auteurs, pour des raisons de mode, consistait à revoir à la baisse les besoins en acide linoléique, et ce, au regard de résultats obtenus à partir d'études menées chez l'animal.

\section{Besoins en acide linoléique (oméga-6)}

Les besoins en acide linoléique, sur le plan biochimique et physiologique, ont été largement explorés chez l'Homme, et ce, à divers âges de la vie :

- des apports en acide linoléique compris entre 0,5 et $1 \%$ de l'énergie totale suffisent à faire disparaître le syndrome de carence cutanée ;

132. Mendy F., Hirtz J., Berret R., Rio B., Serville F., Verger P. (1970) Variation of the ratio of oleic triene--arachidonic acid or C20: 3 omega 9--C20: 4 omega 6--in serum of infants according to age and diet. Arch. Sci. Physiol. (Paris), 24 (3), 279-296. 
- à $1 \%$ de l'énergie totale, le gaspillage énergétique disparaît en atmosphère normale (humidité contrôlée) et thermogenèse réduite (adaptation comportementale), des conditions toutes deux réalisées par les couveuses dans lesquelles sont placés les prématurés ;

- à 1,5\% de l'énergie totale, le rapport C20:3 n-9/C20:4 n-6, commence à baisser ; il atteindra son point le plus bas mesuré au niveau des phospholipides vers 3,5\% de l'énergie totale si les conditions de transformation en dérivés supérieurs de l'acide linoléique (oméga-6 ou $n$-6) ou de l'acide oléique (oméga-9 ou $n$-9) sont remplies ;

- à partir de $5 \%$ de l'énergie totale chez le nourrisson, le taux d'acide arachidonique (C20:4 n-6) stagne et peut même baisser, le plus souvent au niveau des phospholipides, progressivement.

À partir de $5 \%$ de l'énergie totale sous forme d'acide linoléique, jusqu'à $10 \%$ voire davantage, nous rentrons dans la zone d'utilisation pharmacologique de l'acide linoléique. De tels niveaux ont ainsi été recommandés pendant 50 ans, sans jamais disposer de véritables preuves scientifiques, comme traitement préventif de l'athérosclérose, des accidents ischémiques cardiovasculaires et des hypercholestérolémies. La découverte des inhibiteurs de la synthèse du cholestérol, les statines, réellement efficaces, a lentement érodé cette recommandation.

\section{Besoins en acide alpha-linolénique (oméga-3)}

L'acide alpha-linolénique a été partiellement évoqué dans les années 1970 : la matière grasse lactée, base des laits de substitution, supplémentés en acide linoléique ou non, apportait en effet des traces non négligeables d'acide alpha-linolénique, représentant de l'ordre de $0,22 \%$ de l'énergie totale. Pour autant, des auteurs américains, et notamment $\mathrm{S}$. Carson, avaient retrouvé, chez les nourrissons recevant des laits de substitution, des concentrations plus faibles en DHA au niveau du plasma sanguin et des membranes érythrocytaires, qui semblaient liées à des perturbations du développement psychomoteur ou des fonctions visuelles très fines, comparativement aux enfants allaités au lait maternel. Or, les laits de substitution américains utilisés 
se caractérisaient par le fait d'être totalement dépourvus de matières grasses lactiques et, dans le meilleur des cas, d'être pauvres en acide alpha-linolénique en sn-2 (car fabriqués avec de l'huile de soja à biodisponibilité faible). Plus que de réelles perturbations, on observait d'ailleurs essentiellement de légers retards de développement compensés au fil du temps. Une synthèse insuffisante d'acides gras à chaîne très longue (supérieure à 20 atomes de carbone) avait alors été évoquée.

Pourtant, ce n'est qu'à partir des années 1990 que l'étude de l'acide alpha-linolénique fut sérieusement entreprise dans le cadre d'un projet menée sur trois centres hospitaliers (Bordeaux, Caen et Montpellier) par A. Crastes de Paulet, B. Descomps, C. Billeaud, D. Bouglé, P. Sarda et F. Mendy. L'idée de ces médecins : évaluer si l'enrichissement des formules infantiles enrichies en acide alpha-linolénique $(1,95 \%$ des matières grasses totales au lieu de $0,55 \%)$ pouvait améliorer les taux de DHA plasmatiques et érythrocitaires.

Dans cette étude portant sur 88 prématurés de poids moyen à la naissance de 1621 grammes, 31 prématurés recevaient le lait étudié, enrichi en acide alpha-linolénique (oméga-3) ; 32 prématurés recevaient une formule infantile alors commercialisée, pauvre en acide alpha-linolénique; et un groupe contrôle de 25 enfants étaient allaités par leur mère.

Dans les trois laits, la teneur en lipides (essentiellement des matières grasses lactiques) était identique, de même que l'apport en acide linoléique (4,9\% de l'énergie totale). L'acide alpha-linolénique (ALA), dans le lait enrichi étudié, était fourni par de l'huile de colza (biodisponibilité : $66 \%$ versus $27 \%$ dans le soja). Ce choix du colza induisait aussi une supplémentation non négligeable en acide oléique $(+2 \%)$ du lait enrichi comparativement à la formule commercialisée, sachant que cet acide gras représente $18 \%$ de l'énergie totale du lait maternel et seulement $10 \%$ dans les laits de substitution.

Le rapport acide linoléique/acide alpha-linolénique (oméga-6/ oméga-3), corrigé en termes de biodisponibilité, était de 6/1 dans le 
lait enrichi en acide alpha-linolénique (oméga-3) et de 22/1 dans la formule non enrichie.

Les acides gras furent analysés au niveau des triglycérides, des esters de cholestérol, des phospholipides plasmatiques et des phosphatidyléthanolamines érythrocytaires.

Cette publication fut présentée dans sa forme originelle à l'Académie de médecine ${ }^{133,134}$. Puis, plus de cinq publications internationales en exposeront les résultats dans la presse scientifique internationale jusqu'en $2000^{134}$. En effet, les résultats, après 2 à 5 semaines d'alimentation enrichie, s'avéraient remarquables.

L'alimentation avec du lait enrichi en acide alpha-linolénique induit une augmentation significative $(\mathrm{p}<0,0001)$ du DHA au niveau des phospholipides plasmatiques (2,20\% versus $1,54 \%$ dans les laits commerciaux, après 15 jours) et des membranes des globules rouges $(3,14 \%$ versus $2,41 \%)$. Au niveau de ces mêmes membranes des globules rouges, bien que l'on observe des taux supérieurs chez les enfants nourris au sein $(4,08 \%$ contre $3,14 \%$ pour le lait enrichi en acide alpha-linolénique), les valeurs de DHA relevées avec le lait enrichi demeurent dans la zone de normalité du DHA des nourrissons alimentés au lait maternel, malgré le poids de naissance de ces prématurés (1 621 grammes).

Concernant l'acide arachidonique (C20:4 n-6), un oméga-6 dérivé de l'acide linoléique, on note une très faible diminution des concentrations (6,88 \% versus $7,53 \%$ à 15 jours) au niveau des phospholipides plasmatiques et des taux parfaitement normaux au niveau des phosphatidyléthanolamines des globules rouges $(15,78 \%$ versus

133. Crastes de Paulet A., Babin F., Billeaud C., Bouglé D., Sarda P., Mendy F. (1994) Biological effects on premature neonates of a milk formula enriched with alpha-linolenic acid: A multicenter study. Bull. Acad. Natl. Med., 178 (2), 267-273 ; discussion : 273-278.

134. Babin F., Sarda P., Bouglé D, Billeaud C, Mazette S, Combe N, Nouvelot A, Descomps B, Entressangles B., Crastes de Paulet A., Mendy F. (1999) Longitudinal multicentric study of plasma and red blood cell fatty acids and lipids in preterm newborns fed human milk. Biol. Neonate, 75 (5), 285-293. 
$14,86 \%$ dans le lait maternel, soit une différence non significative). La forte augmentation des oméga-3 de la ration ne pénalise donc pas la famille oméga- 6 , alors que l'on aurait pu redouter une concurrence des deux familles liée aux désaturases et élongases communes.

Enfin, les concentrations en acide adrénique (C22:4 n-6) s'avèrent stables chez les enfants alimentés par ces trois types de lait, prouvant que l'on se situe bien dans une zone de régulation (l'acide adrénique fonctionnant comme une « soupape de sécurité » en cas d'excès d'apports).

Parallèlement à cette étude fut réalisée, pour la première fois, une étude sur la filière oléique (18:1 n-9, oméga-9) et nervonique (24:1 n-9, oméga-9) qui donna lieu à une publication dans Lipids, en $1993^{135}$. À partir de la composition des sphingomyélines des globules rouges (ou érythrocytes) fut établi un indice de la maturation de la myéline, chez 28 prématurés de 31,5 semaines d'âge gestationnel. Onze de ces prématurés étaient nourris au lait maternel, 9 reçurent une formule infantile du commerce et 8 une formule enrichie en acide alpha-linolénique. Les résultats montrent que quel que soit le régime, l'évolution après 5 semaines est pratiquement identique : l'acide alpha-linolénique n'interfere pas dans l'élongation de l'acide oléique et la synthèse de l'acide nervonique dans cette étude. L'acide nervonique augmente doucement chez les prématurés, enclenchant le début d'une hausse observée chez les enfants nés à terme : le taux d'acide nervonique augmente progressivement chez les prématurés observés, de 9,9 à 12,8 \% à 37 semaines d'âge postconceptionnel. On sait qu'il progressera par la suite pour atteindre 25,4 \% à l'âge de 5 ans. Dans le cas de l'éclampsie, un accident hypertensif aigu spécifique de la grossesse, ce taux peut être très bas à la naissance, autour de $1 \%$.

Ainsi, au terme de 40 ans de recherche, le métabolisme des acides gras insaturés a été parfaitement exploré, lentement, de 22 semaines

135. Babin F., Sarda P., Limasset B., Descomps B., Rieu D., Mendy F., Crastes de Paulet A. (1993) Nervonic acid in red blood cell sphingomyelin in premature infants: An index of myelin maturation? Lipids, 28 (7), 627-630. 
de gestation à 5 ans de vie. Pourtant, les connaissances issues de ces études n'ont, jusqu'ici, que peu été intégrées dans la réflexion de la biochimie des lipides chez l'adulte, en particulier cette extraordinaire interconnexion, de cette interdépendance, entre les diverses familles d'acide gras. Et ce, même s'il semble qu'elles pourraient être très utiles en tant que modèle d'évolution, rapide et adaptatif.

\section{Évolution des teneurs en acides gras du cerveau avec l'âge gestationnel}
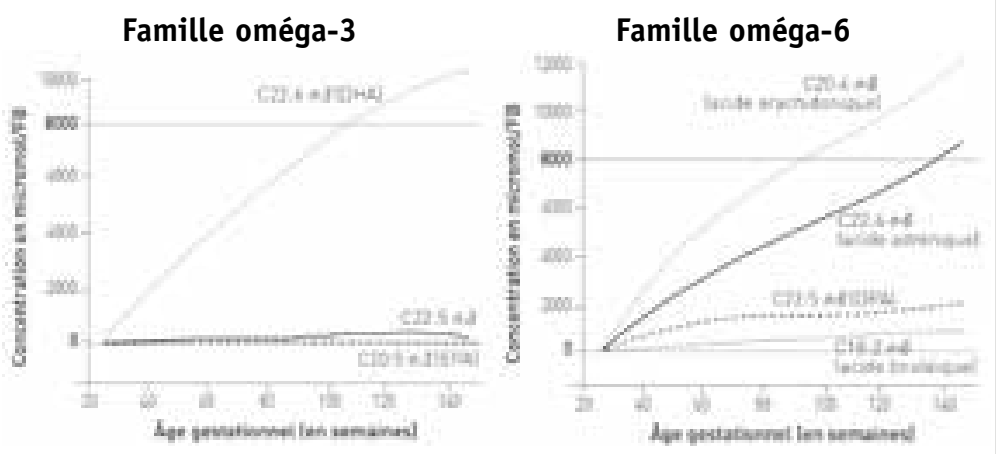

Famille oméga-9

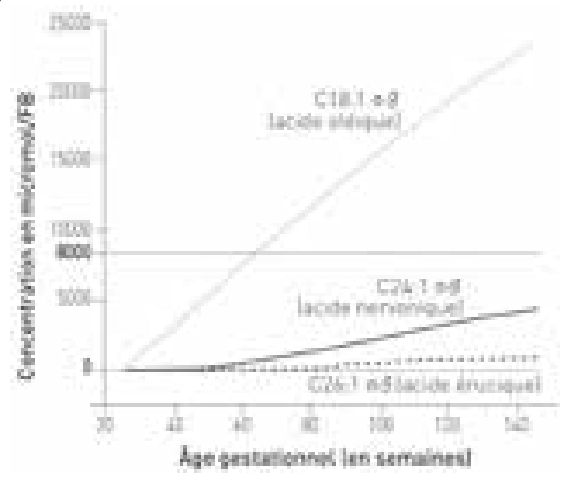




\section{Des besoins énergétiques propres à chaque enfant}

À 2,5-3 ans, le cerveau pèse déjà l'équivalent des trois quarts de son poids à l'âge adulte. À sonné l'heure de l'explosion énergétique, l'âge de la mobilité presque sans limite. Très étudié par E. M. Widdowson (1962 136$)$, B. S. Burke (1959137) et D. W. Sargent (1961), ce sujet fut ensuite quelque peu négligé, jusqu'au travail publié par T. Voortman en $2015^{138}$.

En réalité, les études remarquables des années 1960 avaient parfaitement mis en évidence l'extraordinaire variation des besoins énergétiques d'un individu à l'autre, et ce, malgré le taux de consommation de fait spontanée de l'ordre de 1770 calories dans cette tranche d'âge, pourtant encore très élevé (Institut national d'hygiène, 1960). L'étude de Harvard, menée par Bertha S. Burke en 1959, avait montré que la première déviation standard (ou écart-type) était, dans cette tranche d'âge, de $14 \%$ : ainsi, seuls quelque $65 \%$ des enfants de 3 à 6 ans mangent la ration calorique moyenne de leur âge $\pm 14 \%$, alors que les $45 \%$ restants mangent des rations caloriques qui s'éloignent de plus de $14 \%$ (en plus ou en moins) de la ration moyenne observée. Comme souligné auparavant par le $\mathrm{D}^{\mathrm{r}}$ Elsie M. Widdowson, «dans chaque tranche d'âge, sur 20 garçons, il y a sans exception au moins un enfant qui mangeait au moins deux fois la quantité de calories qu'absorbait un autre de ces enfants".

Chez les filles, les différences s'avèrent encore plus spectaculaires : la valeur de la deuxième déviation standard à 16-17 ans au-dessous de la moyenne est inférieure à la valeur de la première déviation standard au-dessus de la moyenne à 3 ans. Ainsi une fillette de 3 ans

136. Widdowson E. M., Kennedy G. C. (1962) Rate of growth, mature weight and life-span. Proc. R. Soc. Lond. B Biol. Sci., 156, 96-108.

137. Burke B. S., Reed R. B., Van Den Berg A. S., Stuart H. C. (1959) Caloric and protein intakes of children between 1 and 18 years of age. Pediatrics, 24, 922-940.

138. Voortman T., Kiefte-de Jong J. C., Geelen A., Villamor E., Moll H. A., de Jongste J. C., Raat H., Hofman A., Jaddoe V. W., Franco O. H., van den Hooven E. H. (2015) The development of a diet quality score for preschool children and its validation and determinants in the Generation R Study. J. Nutr., 14 5(2), 306-314. 
d'apparence normale peut manger plus qu'une jeune fille de 1617 ans, également d'apparence normale!

Il n'est pas certain que les travaux plus récents de ces dix dernières années puissent, en dehors de l'établissement de « normes centrales » ou de «moyennes » sur des séries de plus de 3000 enfants, résoudre ce problème des variations individuelles, temporaires ou permanentes, qui reste un étonnant problème de régulation.

Finalement, ce sont les courbes de corpulence (poids/taille ${ }^{2}$, où le poids $\mathrm{P}$ est exprimé en kilo et la taille $\mathrm{T}$ en mètre), calculées selon l'indice de Quetelet (du nom d'Adolphe Quetelet, scientifique belge père de cet indice) et établies par 1'Inserm, qui permettent, au fil des mois, de suivre au mieux cette étonnante évolution.

Ainsi cet indice, également présenté comme indice l'adiposité, permet de définir une évolution en trois temps :

- entre 0 et 1 an : l'IMC augmente jusqu'à atteindre un maximum après un délai variable d'un enfant à l'autre : pour le $50^{\mathrm{e}}$ percentile, l'IMC grimpe de 0 à 9 mois; pour le $90^{\mathrm{e}}$ percentile de 0 à 1 an. À 1 an, l'IMC moyen est de $17,5 \mathrm{~kg} / \mathrm{m}^{2}$;

- de 1 à 6 ans : l'IMC diminue pour atteindre son point le plus bas à 6 ans, avec une valeur moyenne de $15,6 \mathrm{~kg} / \mathrm{m}^{2}$. Cette diminution, corrélée à l'explosion motrice de cette tranche d'âge, est plus constatée que réellement comprise sur le plan de sa régulation ;

- à 6 ans et plus : l'IMC va remonter de nouveau pour atteindre la valeur de 21 à l'âge de 20 ans et, par la suite, progressivement grimper jusqu'à un maximum de 25,8 à 65 ans, avant d'entamer un déclin progressif pour atteindre 23,5 à 85 ans $\left(90^{\mathrm{e}}\right.$ percentile $=$ $28 \mathrm{~kg} / \mathrm{m}^{2}$ à 85 ans).

La phase de mobilité maximum entre 2,5 et 6 ans s'avère donc incroyablement régulée, même si l'on ne connaît pas bien les mécanismes de cette régulation. L'observation de l'enfant, en tant qu'être unique, et non comme élément d'une cohorte statistique, est donc indispensable. 


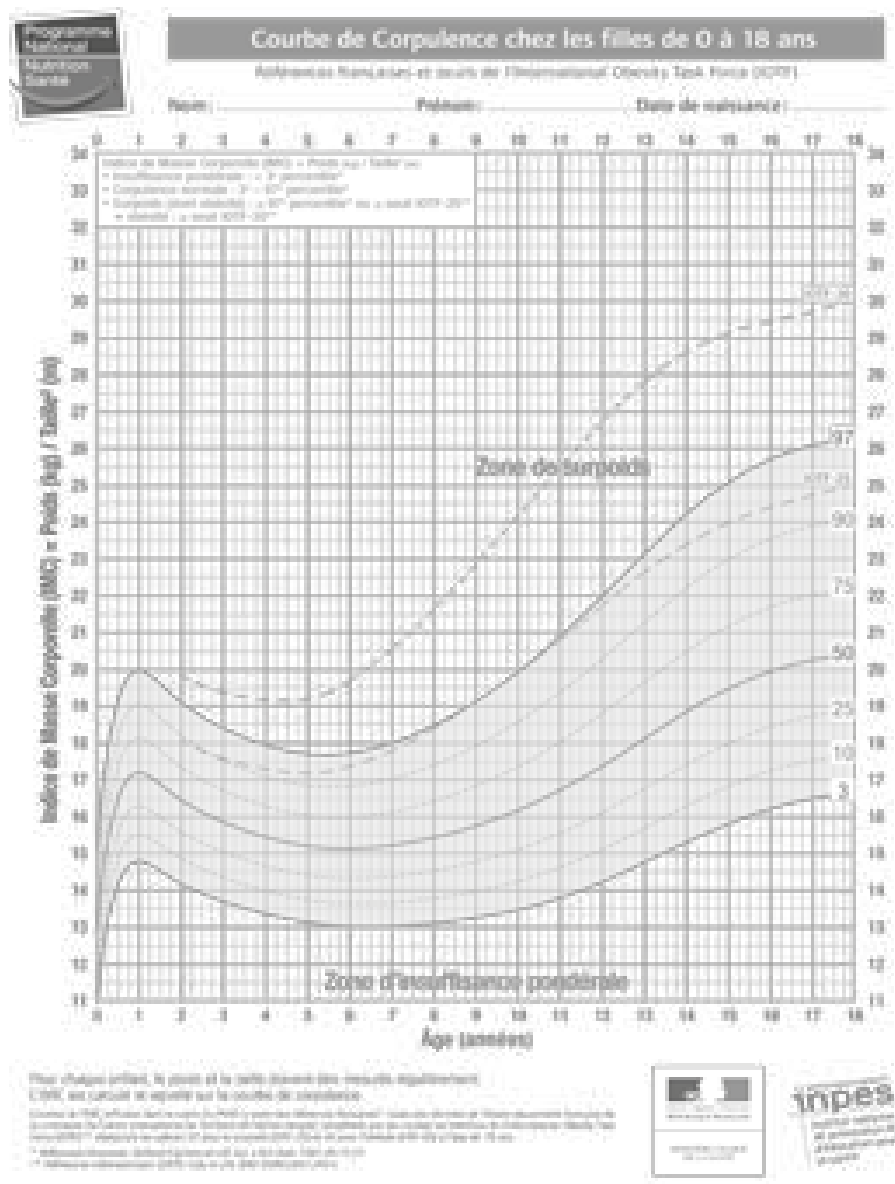

Une question fondamentale se pose : le supplément énergétique que l'enfant réclame pour compenser des dépenses énergétiques impressionnantes doit-il, suivant la mode, être uniquement glucidique ? Ou également lipidique ? Tout laisse à penser que $40 \%$ des calories amenées par ce supplément devraient être d'origine lipidique (matières grasses lactiques par exemple), afin de ne pas trop s'éloigner (oublier) des performances des ratios entre protéines, glucides et lipides de l'aliment de référence, le lait maternel. 


\section{Du danger des régimes et des modes}

À chaque époque sa mode... et son ennemi public : après les régimes " pauvres en lipides » est venu le temps des régimes " pauvres en glucides ». Il est difficile de faire comprendre le danger des diététiques alternatives, tant le marketing scientifique mené autour de l'étude des sept pays (Seven Countries Study, conçue par le physiologiste américain Ancel Keys), parangon de ces premières études épidémiologiques, a été puissant. L'introduction d'onces de vérité, malheureusement complexes, s'avère très difficile dans un marketing forcément réducteur.

Devant la nécessité, enfin aveuglante, d'évoluer, la formulation de transition la plus subtile fut ce message : "Choose a diet that is low in saturated fat and cholesterol and moderate in total fat " ("Choisir un régime qui est faible en graisses saturées et en cholestérol, et modéré en matières grasses totales »), issu des recommandations nutritionnelles et de santé à destination des Américains, publiées en 2000, comme le commente L. B. Dixon dans un article paru en $2001^{139}$. C'est ainsi que la puissance du marketing finit par influencer l'alimentation de l'enfant de 3 à 6 ans, au mépris de toutes les connaissances scientifiques accumulées.

Mais pour un pédiatre américain, Ronald Krause, un chemin inverse restait possible. En s'inspirant de l'alimentation spontanée du nourrisson et de l'enfant, il était peut-être intéressant de proposer une voie alternative : "Le danger des régimes riches en glucides (hydrates de carbone), pauvres en lipides. » Sur le plan métabolique, ces régimes sont la meilleure source d'acides palmitique et oléique endogènes, entraînant une hausse du taux de sécrétion des VLDL, du rapport cholestérol total/ cholestérol HDL ( " mauvais/bon ») ou du rapport entre le cholestérol total (cholestérol VLDL + cholestérol IDL + cholestérol LDL) et le

139. Dixon L. B., Ernst N. D. (2001) Choose a diet that is low in saturated fat and cholesterol and moderate in total fat: Subtle changes to a familiar message. J. Nutr., 131 (2S-1), 510S-526S. 
cholestérol HDL (voir notre leçon dédiée au cholestérol). En réalité, c'est toute une lipogenèse de novo anormale qui est enclenchée.

Mais le danger de tels régimes ne s'arrête pas là : on observe également l'augmentation des «small » LDL (les LDL les plus athérogènes). La variation de taille des particules LDL a permis de mettre en évidence l'existence de deux phénotypes au sein de la population : - un phénotype B, associé aux particules LDL plus denses, petites et oxydables ( small » LDL), des taux de triglycérides élevés et une consommation élevée de lipides, est amélioré par un régime pauvre en graisses saturées ;

- un phénotype $\mathrm{A}$, où les particules LDL sont moins oxydables, les triglycérides bas et le HDL ( «bon cholestérol ») élevé.

Les sujets qui expriment le phénotype B avec une consommation élevée de lipides sont favorisés par un régime pauvre en graisses saturées et en cholestérol.

En revanche, les sujets qui expriment le phénotype A ne montrent pas, avec un régime abaissé en matières grasses, de diminution de l'Apo B mais une augmentation des «small " LDL, une diminution des HDL2b les plus protectrices et affichent un risque cardiovasculaire pratiquement doublé. Cela s'avère particulièrement vrai pour une sous-fraction du phénotype $A$, particulièrement sensible aux teneurs en glucides du régime, qui se transforme en phénotype $B$ en passant d'un régime à teneur lipidique élevée à un régime à teneur lipidique faible et à teneur glucidique élevée.

Les conséquences de la dérive vers des régimes à teneur lipidique faible et à teneur glucidique élevée sont impressionnantes :

- à l'âge pédiatrique, pour le moment, le phénotype $\mathrm{B}$, exposé et qui tirerait bénéfice d'un régime pauvre en graisses saturées et cholestérol, concerne 5 à $10 \%$ des sujets ;

- à l'âge adulte, le régime "pauvre en lipides, riche en glucides " serait à lui seul responsable de $36 \%$ de phénotypes B supplémentaires (les anciens phénotypes $\mathrm{A}$ soumis à un régime pauvre en lipides et riche en glucides). 
Deux conclusions s'imposent :

- la nutrition de l'enfant de 0 à 2 puis 6 ans doit être dominée à la fois par des données traditionnelles, empiriques et d'observation, par les apports de plus en plus riches de la biochimie et de la physiologie humaine, et non par le marketing des produits pour adultes; - plutôt que de vouloir, sans cesse, tenter d'imposer les modèles de consommation de l'adulte, entre autres pour créer très tôt des habitudes de consommation, il semble que le chemin inverse serait plus rentable, pour au moins $36 \%$ de la population.

À ce stade, une question fondamentale se pose : pour améliorer les $10 \%$ de la population à risque (phénotype B), doit-on, sur le plan national, suggérer un régime qui s'avère dangereux pour $36 \%$ de la population (la sous-fraction de phénotypes A qui va basculer en phénotype $\mathrm{B}$ du fait d'un régime pauvre en lipides et riche en glucides) ? 


\section{" À retenir}

Durant les quatre derniers mois de vie utérine, le bébé grossit de 2,5 kg et grandit de $40 \mathrm{~cm}$, ce qui nécessite une énorme quantité d'énergie. Les lipides, et notamment un acide gras particulier (l'acide palmitique), fournissent la majeure partie de cette énergie.

L'acide palmitique (C16:0) représente le carrefour, la plate-forme énergétique $d u$ " bébé » in utero.

Cette étonnante plate-forme énergétique est corégulée avec son dérivé, l'acide palmitoléique (C16:1 $\omega 7$ ).

Entre 0 et 2 ans, le lait maternel est l'aliment de référence. Dans les premiers jours, le nourrisson a besoin de près de 5 grammes de lipides par kilo de poids corporel. Vingt ans plus tard, à l'âge adulte, ce besoin est de l'ordre de 1 à 1,25 gramme de lipides par kilo et par jour.

$\grave{A}$ la naissance, le cerveau pèse $25 \%$ du poids d'un cerveau adulte ; $a$ mois, $50 \%$; à 2,5 ans, $75 \%$.

Les seuls acides gras indispensables sont les acides linoléique (oméga-6) et alpha-linolénique (oméga-3). Ce n'est que dans les années 1990 que l'on montra que les laits infantiles destinés aux prématurés devraient être davantage enrichis en acide alpha-linolénique.

Les besoins énergétiques entre 2 et 6 ans sont importants. Les moyennes ne doivent pas faire oublier l'énorme variabilité interindividuelle, soustendue par des mécanismes de régulation encore mal connus.

Plaquer sur des enfants des messages diététiques dictés par des effets de mode (moins de graisses, plus de glucides, etc.) s'avère dangereux et peut faire basculer certains individus normaux dans des situations pathologiques. 
